\title{
RIKEN Accelerator-driven compact neutron systems
}

\author{
Yoshie Otake, ${ }^{1, *}$ \\ ${ }^{1}$ RIKEN Center for Advanced Photonics, Neutron Beam Technology Team, RIKEN 351-0198 Saitama, Japan
}

\begin{abstract}
RIKEN Accelerator-driven compact neutron source, RANS, has been operational since 2013. There are two major goals of RANS research and development. One is to establish a new compact low energy neutron non-destructive evaluation system on-site of floor-standing type for industrial use. Another goal is to invent a novel transportable compact neutron system for the preventive maintenance of large scale construction such as a bridge. The low energy transmission imaging, neutron diffractometer, small angle scattering instruments, fast neutron transmission imaging, fast neutron back scattering imaging, neutron induced prompt gamma-ray analysis and neutron activation analysis are available with RANS. To solve the lack of nuclear data to develop compact neutron sources, we have created new function which gives neutron spectrum from the $9 \mathrm{Be}+\mathrm{p}$ reaction with protons of energy below $12 \mathrm{MeV}$. For further compact neutron system, RANS-II is now ready to generate neutrons in the RANS experimental hall with individual shielding system. The novel proton accelerator tube and $500 \mathrm{MHz}$ solid state high-frequency amplifier for RANS-III, of a transportable neutron system, are now ready to be developed in RIKEN.
\end{abstract}

\section{Introduction}

Accelerator-driven compact neutron sources have attracted much attention not only in neutron and X-ray fields but also in fields such as materials development aimed at significant weight reduction in automobiles and other transportations, cancer therapy, and non-destructive inspection technology. A neutron beam has unique characteristics such as high penetration power for penetrating into steels and metals and high sensitivity for light elements (e.g., hydrogen, lithium, and boron), thus making the neutron beam an ideal probe for material development. RIKEN has developed an acceleratordriven compact neutron source, RIKEN acceleratordriven compact neutron source (RANS) with $7 \mathrm{MeV}$ proton and the beryllium target, as shown in Fig. 1 [1]. There are two major goals of RANS research and development. One is to establish a new compact floorstanding type low-energy neutron system for industrial use. This is RANS-II which is becoming one of the typical model of this type. Another goal is to invent a novel transportable compact neutron system for the preventive maintenance of large-scale construction, such as bridges and airports, using fast neutron whose energy is above $500 \mathrm{keV}$.

For the accelerator driven compact neutron source development, the discrepancy of the neutron spectrum and its angular distribution between any of nuclear data of $9 \mathrm{Be}$ $+p$ reaction and experimental results, is now widely recognized especially for the lower energy of proton. To solve this issue to develop compact neutron sources, we have created new formalism which gives neutron spectrum from the $9 \mathrm{Be}+\mathrm{p}$ reaction for protons of energy below $12 \mathrm{MeV}$ [2]. Here about the formalism will be explained in next chapter, and the RANS spectrum for various kinds of moderator will be shown based on it. Some results of thermal and fast neutron imaging, neutron scattering with RANS and further compact neutron system will be also explained.

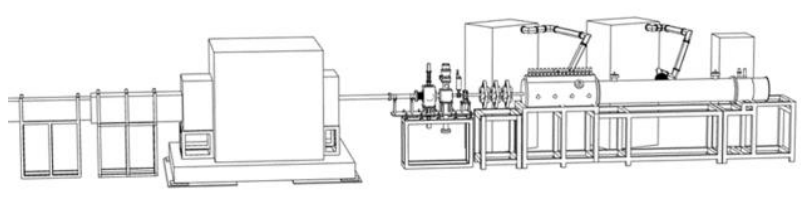

Fig.1 RIKEN Accelerator-driven compact Neutron Source, RANS. Proton linear accelerator of $7 \mathrm{MeV}$ (right), target station (center) with Be target and neutron beamline (left)

\section{Formalism of neutron spectrum of $9 \mathrm{Be}+\mathrm{p}$ reaction for compact neutron source [2]}

The measured radiation background around RANS and the neutron spectrum of RANS did not agree with the designed value which was studied based on Monte Carlo simulation and nuclear data base [3]. We have developed new formalism, a function, to give the total neutron production cross section, angular distribution, and energy spectrum via the $9 \mathrm{Be}+\mathrm{p}$ reaction by fitting experimental data to characterize compact neutron sources with thick

\footnotetext{
* Corresponding author: yotake@riken.jp
} 
Be targets bombarded by protons with energy below 12 $\mathrm{MeV}$ [2].

To derive the neutron production with a certain thick target, a computer program with a Monte Carlo calculation was developed by incorporating the function and the energy loss in the target material calculated by the SRIM code [4]. The program requires only proton energy and the target thickness as input parameters. The calculation was carried out using the $\mathrm{C}++$ programming language and the ROOT framework [5]. The function was examined at RANS as shown in Fig.2. The ${ }^{115} \operatorname{In}(\mathrm{n}$, $\left.\mathrm{n}^{\prime}\right)^{115 \mathrm{~m}} \mathrm{In}$ reaction rates calculated using GEANT4 with source neutrons given by both the function and ENDF/BVII.0 were compared with that measured at RANS to evaluate the neutron spectrum above $1 \mathrm{MeV}$. The function slightly overestimated the measurement by $14 \%$ and the calculation with ENDF/B-VII.0 underestimated by $35 \%$. It was concluded that the function can be applied in compact neutron source designs.

Now, for the design of new compact neutron sources with beryllium target and proton whose energy is less than 12 $\mathrm{MeV}$, the program with this function is available. The neutron spectrum and angler distribution will be obtained when the target thickness, and proton energy are given.

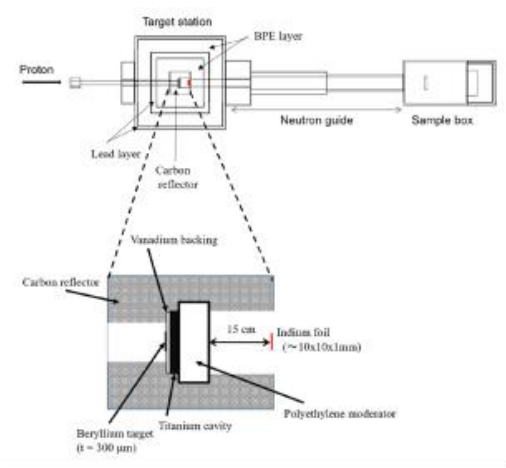

Fig.2 Schematic view of the neutron measurement with ${ }^{115} \operatorname{In}\left(\mathrm{n}, \mathrm{n}^{\prime}\right)^{115 \mathrm{~m}} \mathrm{In}$ reaction at RANS [2]

\section{RANS neutron spectrum moderator change system}

Neutron non-destructive visualization with slow and fast neutron, neutron engineering diffraction for microstructural analysis of iron and steel samples, elemental analysis with neutron induced prompt gammaray activation analysis are the major subjects that we are requested to perform with RANS based on the strong request from the users of such field as industry, public work. RANS can offer users optimized neutron system, by introducing different kinds of moderators. Users can choose suitable moderator for each experiments.

The neutron spectrum at the exit of target station with different moderator condition of case 1, 2, 3, 4 which are listed in Table.1, are shown in Fig.3. The neutron spectrum from the beryllium target [6] is given by the function which was explained in the previous session [2].

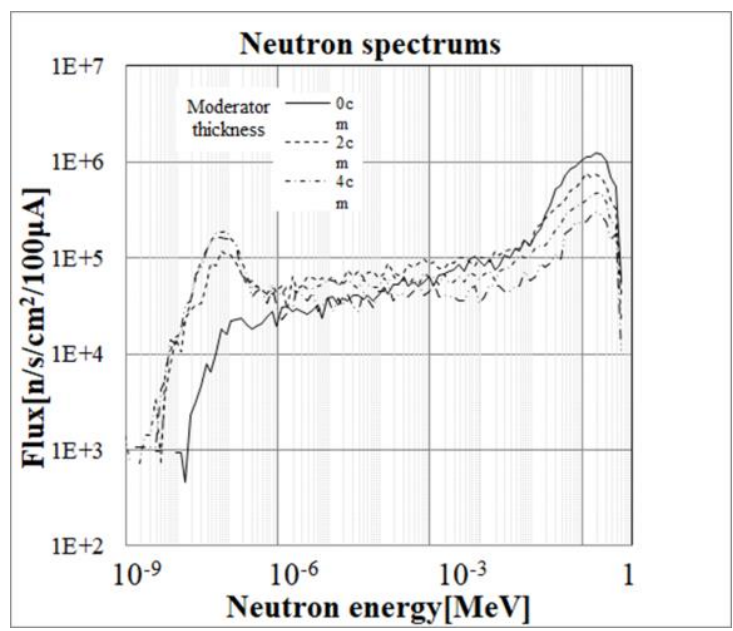

Fig.3 Neutron spectrums at the exit of target station of RANS, about $1.5 \mathrm{~m}$ down from the target for different kinds of polyethylene moderators.

The moderator exchange system is available so that the users can chose suitable moderator for their measurements. They can choose one among several kinds of the neutron energy spectrum and pulse characteristics by changing the different types of the moderator from fast neutron to cold neutron. The table 1 shows the different types of moderator of RANS in 2019. The moderator choice is as following. Case 1, without moderator for fast neutron experiment, case 2 to case 4 are thermal neutron moderators with polyethylene of different thickness, $2 \mathrm{~cm}, 4 \mathrm{~cm}, 6 \mathrm{~cm}$, respectively. Case 5 is the decoupled moderator with $2 \mathrm{~cm}$ polyethylene surrounded by B4C rubber. Case 6 is cold moderator which is now under upgrade development with the cooperation with the group of J-PARC center [7]. The neutron spectrums of type 1 to 4 are shown in Fig. 4. The fast neutron flux decreases according to the thickness of moderator increasing.

Table 1. RANS different types of moderator

\begin{tabular}{|c|l|l|}
\hline & Moderator material & Thickness, and type \\
\hline 1 & None & \\
\hline 2 & $\begin{array}{l}\text { Polyethylene (thermal } \\
\text { moderator) }\end{array}$ & $2 \mathrm{~cm}$ coupled \\
3 & & $4 \mathrm{~cm}$ coupled \\
4 & & $6 \mathrm{~cm}$ coupled \\
5 & & $4 \mathrm{~cm}$ decoupled \\
\hline 6 & $\begin{array}{l}\text { Mesitylene (cold } \\
\text { moderator) }\end{array}$ & up-grade development \\
\hline
\end{tabular}

From Fig. 3 there is no difference of the neutron flux around $50 \mathrm{meV}$ among case $3,4 \mathrm{~cm}$, and case $4,6 \mathrm{~cm}$, but the fast neutron flux above $1 \mathrm{MeV}$ with case 4 is more than $50 \%$ less than case 3 , so that the users can perform the scattering experiment with less background caused by fast neutron by choosing case 5 moderator.

Fig. 4 shows four kinds of the moderators from case 2 to case 5 . The right one shows the decoupller, B4C rubber of decoupled moderator, Case 5. 


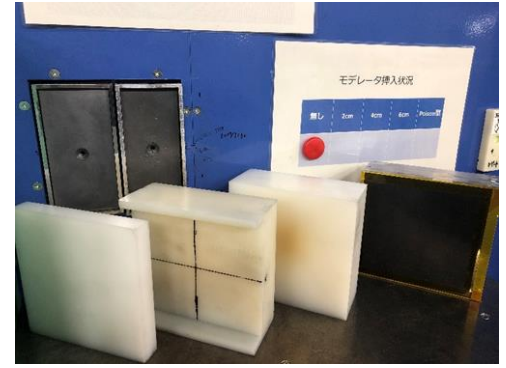

Fig.4 Four kinds of thermal moderator of RANS. Case 2 to case 5 from left to right.

For the coupled moderator case 2 are designed based on the pulse width measurements of RANS with diffraction peak evaluation by changing the proton pulse width and sample thickness [8]. Further shorter neutron pulse, thermal decoupled moderator case 5 is designed and now has being estimated by the RANS diffraction experiment.

\section{Capabilities of RANS, compact neutron source}

\subsection{Neutron scattering instruments}

From 2013, the number of neutron scattering instruments are increasing at RANS. For example, the neutron low energy imaging starts in 2013, the visualization of the water movement which is related to the painted steel corrosion has been observed and new analytical method has been developed [9]. The neutron engineering diffraction method has been developed from 2014 [8], and the diffractometer are now ready for users as shown in Fig.5.

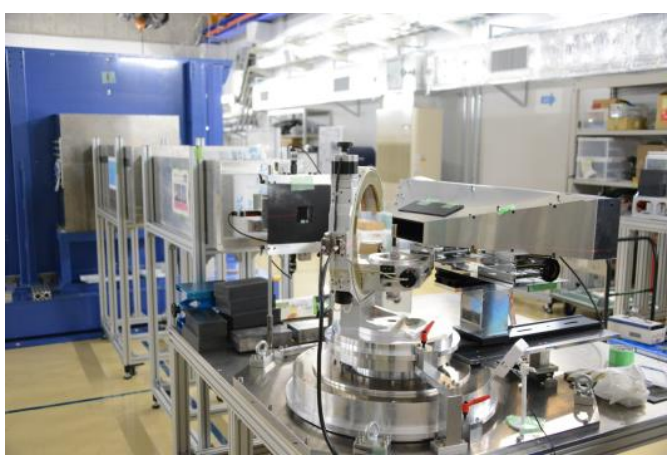

Fig.5 Diffractometer of RANS without shielding

The retained austenite volume fraction evaluation of RANS diffractometer agrees with that of J-PARC Takumi-beamline measurement within 1\% accuracy [10]. Texture evaluation of IF steel samples are measured and compared with J-PARC TAKUMI results and HIPPO results [11]. One diffraction pattern measurement of the iron sample with $1 \mathrm{~cm}^{3}$ volume takes about one to three minutes by RANS. Small angle neutron scattering instrument also has been developed by Ibaraki University. Some results agree with those of J-PARC iMATERIA [12].

\subsection{RANS-II}

For further development towards the floor-standing compact system for industrial use, almost all these instruments are expected to be operational on-site. RANSII succeed to generate neutron with lithium target system as shown in Fig. 6. RANS-II is the floor standing type model for on-site use, and at the same time, it is the prototype of the transportable neutron source [13]. Since July 2019, RANS and RANS-II are operational independently; each has their own interlock safety system.

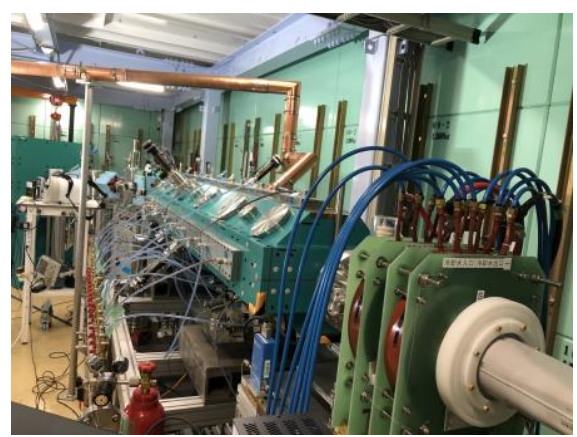

Fig.6 RANS-II with ECR ion source, $2.49 \mathrm{MeV}$ proton linac RFQ, and target station.

\subsection{Infrastructure non-destructive test techniques}

The deterioration of transportation infrastructure is a serious yet common problem around the world. Most large structures, such as bridges, tunnels, and elevated roads, are made of concrete and steel. In 2018, and 2019, large bridges collapsed in Italy and Taiwan [14]. It is becoming increasingly important to establish new inspection techniques.

We have developed back scattered neutron imaging method, novel method to visualize the degradation under the pavement, degradations of the floor-slab of the bridges. The fast neutrons penetrate through more than $20 \mathrm{~cm}$ concrete or asphalt layer of pavement. By choosing the proper timing and the energy of the back scattered neutron, the visualization of the water or air hole in the floor slab behind the first layer of the pavement [15].

We also have successfully developed the salt concentration distribution evaluation method with neutron induced prompt gamma-ray activation analysis for thick concrete slab with RANS [16], as shown in Fig. 
7. The different depth of the salt existence in the concrete can be distinguished.
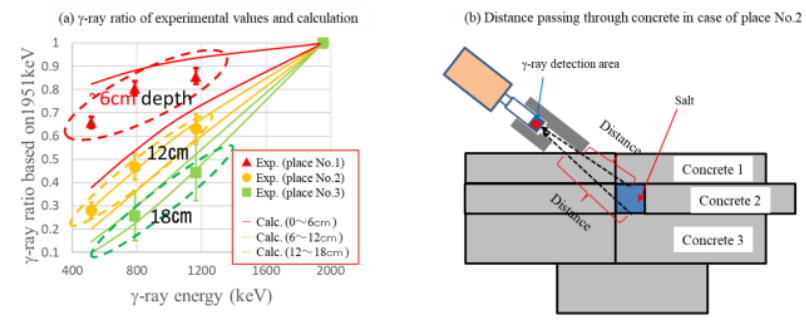

Fig.7 Salt distribution evaluation method with PGAA

\section{Summary}

Compact neutron systems have been developed in RIKEN with proton linac.

The low energy transmission imaging, neutron engineering diffractometer, small angle scattering instruments, fast neutron transmission imaging, fast neutron back scattering imaging, neutron induced prompt gamma-ray analysis and neutron activation analysis are available with RANS.

The up-grade of the RANS target station including cold neutron source optimization is planning in 2020 .

For the transportable, the development of RANSIII, which is expected to be a transportable neutron system is now started. Smaller proton linac with 500 $\mathrm{MHz}$ is designed [17].

\section{Acknowledgment}

This work was partially supported by the Council for Science, Technology and Innovation (CSTI), the CrossMinisterial Strategic Innovation Promotion Program (SIP), "Infrastructure Maintenance, Renovation and Management" (funding agency: JST), and the Photon and Quantum Basic Research Coordinated Development Program from the Ministry of Education, Culture, Sport, Science, and Technology, Japan. It was also supported by JSPS KAKENHI, Grant Numbers 25289265 and 25420078. The authors would like to thank the Iron and Steel Institute of Japan (ISIJ) Research Group for their beneficial assistance.

\section{References}

1. Y. Otake " A Compact Proton Linac Neutron Source at RIKEN", "Applications of Laser-Driven Particle Acceleration", eds. Paul Bolton, Katia Parodi, Jörg Schreiber, June 5 pp.291-314 (2018) CRC Press
2. Yasuo Wakabayashi, Atsushi Taketani, Takao Hashiguchi, Yoshimasa Ikeda, Tomohiro Kobayashi, Sheng Wang, Mingfei Yan, Masahide Harada, Yujiro Ikeda, and Yoshie Otake: "A function to provide neutron spectrum produced from the $9 \mathrm{Be}+\mathrm{p}$ reaction with protons of energy below $12 \mathrm{MeV}[\mathrm{J}] . "$, Journal of Nuclear Science and Technology Vol. 55 (2018.3) pp.859-867

3. Baolong Ma, Yoshie Otake, Sheng Wang, Hideyuki Sunaga, Yutaka Yamagata, Atsushi Taketani, Huasi $\mathrm{Hu}$, Qinggang Jia, Guang $\mathrm{Hu}$, Unico Bautista: "Shielding design of a target station and radiation dose level investigation of proton linac for a compact accelerator-driven neutron source applied at industrial sites", Applied Radiation and Isotopes Vol.137 (2018.7) pp. 129-138

4. Ziegler JF, Biersack J, Littmark U. The stopping and range of ions in solids. New York: Pergamon Press; 1985.

5. ROOT a Data Analysis Framework [internet]: CERN; c2014-2018. Available from: https://root.cern.ch

6. Y.Yamagata, K.Hirota, J.Ju, S.Wang, S.Morita, J.Kato, Y.Otake, A.Taketani, Y.Seki, Y.Yamada, H.Ota, U.Bautista, Q.Jia : " Development of a neutron generating target for compact neutron soruces using low energy proton beams, "Journal of Radioanalytical and Nuclear Chemistry 305, Issue 3 (2015.9) pp787-794

7. B.Ma UCANS8 presentation

8. Y. Ikeda, et al "Prospect for application of compact accelerator-based neutron source to neutron engineering diffraction", Nucl. Instr. Meth. A 833 (2016) pp 61-67

9. Atsushi Taketani, et al. "Visualization of water in corroded region of painted steels at a compact neutron source" ISIJ International vol.57 N.1 (2017) pp.155-161

10. Y. Ikeda, M. Takamura, T. Hakoyama, Y. Otake, M. Kumagai, and H. Suzuki, "On-site Measurement Technique of Retained Austenite Volume Fraction by Compact Neutron Source RANS", Tetsu to Hagane, Vol.104 (2018) pp.18-pp.24

11. P.G. Xu, UCANS8 presentation

12. S.Koizumi, et al. "A Variety of Small-angle Neutron Scattering Instruments Available in Tokyo Area, Japan - Complimentary Use of Accelerator and Reactor-” JPS Conf. Proc. 25, 011004 (2019)

13. T.Kobayashi et al, UCANS8 presentation

14. https://edition.cnn.com/2019/10/01/asia/taiwanbridge-collapse-intl-hnk-scli/index.html

15. Y. Ikeda, Y. Otake, and M. Mizuta: nondestructive measurement for water and voids in concrete with compact neutron source", Plasma and Fusion ResearchVol.13 (2018.3) pp.2406005-1-5 DOI: 10.1585/pfr.13.2406005

16. Y.Wakabayashi UCANS8 presentation 
17. S.Ikeda, Y.OTAKE, T.Kobayashi, and N.Hayashizaki, " Design of $500 \mathrm{MHz}$ RFQ linear accelerator for a compact neutron source, RANSIII ", Nucl. Instr. Meth. B 461 (2019) pp 186-190 